\title{
Seismic response of the geologic structure underlying the Roman Colosseum and a 2-D resonance of a sediment valley
}

\author{
Peter Moczo $\left({ }^{1}\right)$, Antonio Rovelli $\left({ }^{2}\right)$, Peter Labák $\left({ }^{1}\right)$ and Luca Malagnini $\left({ }^{2}\right)$
$\left({ }^{1}\right)$ Geophysical Institute, Slovak Academy of Sciences, Dúbravská cesta, Bratislava, Slovak Republic \\ $\left.{ }^{2}\right)$ Istituto Nazionale di Geofisica, Roma, Italy
}

\begin{abstract}
The seismic response of the geologic structure beneath the Colosseum is investigated using a two-dimensional modeling for a vertically incident plane $S H$ wave. Computations indicate that the southern part of the Colosseum may be exposed to a seismic ground motion with significantly larger amplitudes, differential motion and longer duration than the northern part, because the southern part of the Colosseum is underlain by a sedimentfilled valley created by sedimentary filling of the former tributary of the River Tiber. A 2-D resonance may develop in the valley. Unlike the previous theoretical studies on 2-D resonance in sediment-filled valleys, an effect of heterogeneous valley surroundings on the resonance is partly investigated. A very small sensitivity of the maximum spectral amplifications connected with the fundamental and first higher modes to the presence of a horizontal surface layer (with an intermediate velocity) in the valley surroundings is observed in the studied models.
\end{abstract}

Key words Roman Colosseum - seismic waves seismic response - site effects - 2-D resonance finite-difference method

\section{Introduction}

The Colosseum, which took its name from the colossal statue that once stood nearby, is one of the most famous Imperial monuments of Rome (fig. 1). The Emperor Vespasiano (6979 A.D.) began construction of the amphitheater, but the Emperor Tito (79-81 A.D.) oversaw the completion of it. Amphitheaters had an important place in the life of ancient Romans; the decision of the Emperor Vespasiano to raze the Domus Aurea, built by the previous Emperor (very unpopular Nero), and to build a monument for popular recreation, was a clear sign of his political intent. The Colosseum was built on the area previously occupied by the artificial lake adorning Nero's residence (Stagnus Neronis).

Various reasons have caused the Colosseum present deterioration. Marble stands and seats were quarried by Renaissance popes and the Roman aristocracy for use in new constructions, the interior was wildly modified; natural hazards as fire, lightening, soil settlement, and earthquakes have also contributed heavily. The outside of the arena is still well preserved around three quarters of its circumference: $50 \%$ of the outer wall is missing on the southern side, and part of the interior wall has been rebuilt.

The Italian earthquake catalogue, spanning more than two thousand years, includes several strong earthquakes in the Central Apennines which were felt in Rome with an intensity up 


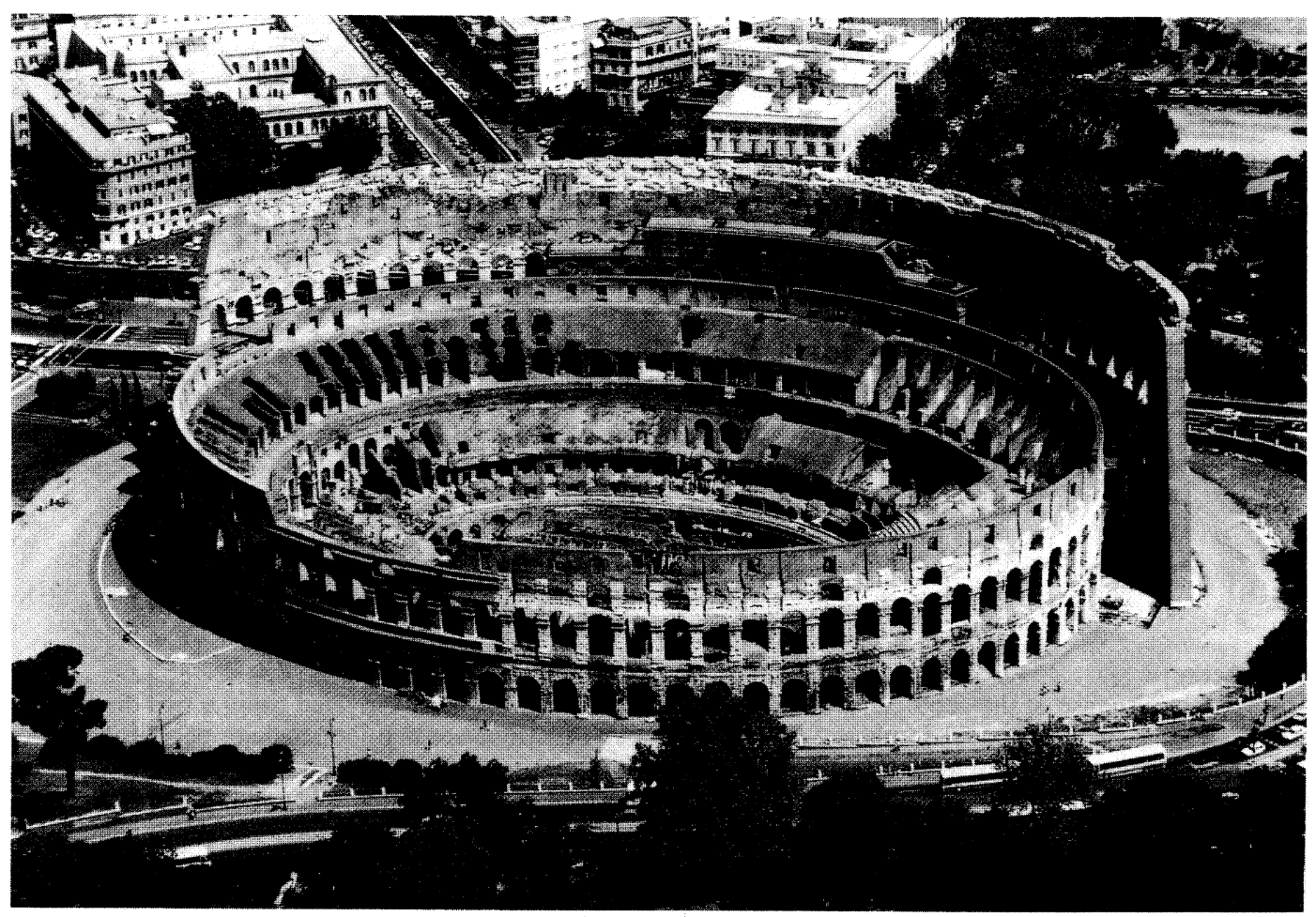

Fig. 1. Aerial picture of the Roman Colosseum. The southern part of the outer wall is missing.

to the VII degree MCS (see Molin and Guidoboni, 1989). The estimated epicentral distances of the strongest earthquakes were about $100 \mathrm{~km}$. Paleoseismic studies (Pantosti et al., 1992) show that the magnitude of these earthquakes might have been 7 or more. The earthquakes of 801, 1348, and 1703 caused huge damage in the city of Rome. Even though we lack reliable and detailed information on the damage produced by the single event, several inscriptions and chronicles cite reconstruction and restorations of the Colosseum ordered to repair seismically induced damage. A wider discussion on earthquakes possibly responsible for major damage to the monument can be found in Funiciello et al. (1995).

A less known fact is the very specific geologic condition beneath the Colosseum. While the northern part of the Colosseum is underlain by an almost horizontal layer of Pleistocene continental deposits, there is a much softer sedimentary filling of a former tributary of the River Tiber beneath the southern part of the Colosseum. The soft tributary deposits create a well confined and relatively deep sedimentfilled valley.

As predicted by the theory and actually observed, the surface ground motion in the sediment-filled valleys may be significantly amplified and prolonged by laterally propagating local surface waves (Bard and Bouchon, 1980; Seo and Kobayashi, 1980; Frankel et al., 1991; Kagawa et al., 1992; Phillips et al., 1993; Frankel, 1993) or by resonance phenomena involving a whole sediment body (King and Tucker, 1984; Bard and Bouchon, 1985). On the other hand, the steep valley slope between contrasting materials represents a strong lateral discontinuity which 
may cause a significant differential motion on its softer side (Moczo and Bard, 1993).

Hence we may expect a large variability of the seismic ground motion within the Colosseum area. We may suspect at least the following factors that might have contributed to the damage during past earthquakes: a) the fact that the Colosseum spans over the strong lateral discontinuity and the related large differential motion on the valley side; b) the large amplification and longer duration of the ground motion beneath the southern part of Colosseum due to the sedimentary valley.

A good understanding of the specific damage pattern may be probably achieved by a complex analysis that will include a realistic excitation, a three-dimensionally inhomogeneous model of the geologic conditions and a soil-Colosseum structure interaction. This is not an easy task and, very likely, it will be solved only step by step.

Currently, only limited data are available on the underlying geologic structure. In this firststep preliminary study we use a two-dimensional model of the geologic structure (without the Colosseum) to investigate the seismic response to a vertically incident $S H$ plane wave.

We believe that even such a simple $S H$ study may provide a useful preliminary estimate of the site effect within the Colosseum area. Moreover, it will be valuable to compare the results obtained from the present $S H$ study and a future study which will include a realistic input signal and a 3-D model.

\section{Model and methods of computation}

\subsection{Model}

Figure 2 shows the geometry and mechanical parameters of the computational model of the local geologic conditions in the north-south direction. The location of the Colosseum structure is also indicated. The model was compiled in the Istituto Nazionale di Geofisica, Rome, by Renato Funiciello and Fabrizio Marra who used all available direct and indirect geophysical and geological data. More geologic wells and geophysical surveys would be necessary to
MECHANICAL PARAMETERS OF THE MODELS

\begin{tabular}{|c|c|c|c|c|c|c|}
\hline & \multicolumn{3}{|c|}{ VELOCITY } & \multirow{2}{*}{$\begin{array}{c}\text { DENSITY } \\
{\left[\mathrm{kg} / \mathrm{m}^{3}\right]}\end{array}$} & \multicolumn{2}{|c|}{ Q FACTOR } \\
\hline & M1 & {$[\mathrm{m} / \mathrm{s}]$} & M2 & & M1 & M2 \\
\hline 1 & & 400 & & 1900 & & \\
\hline 2 & 100 & & 200 & 1800 & 15 & 7 \\
\hline 3 & & 400 & & 1900 & & \\
\hline 4 & & 800 & & 2050 & & \\
\hline
\end{tabular}
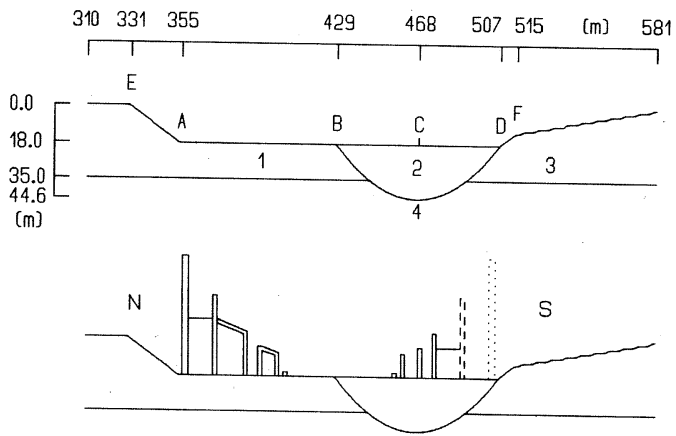

Fig. 2. Geometry and mechanical parameters of models of the local geologic conditions in the northsouth direction. Two extreme models M1 and M2 with different velocities and quality factors in the valley were considered for the same geometry in order to obtain upper and lower estimates of seismic response. The position of the Colosseum is schematically indicated. The northern and southern parts of the Colosseum overlie the A-B and B-C-D segments, respectively. Only part of the computational model is shown.

check details of this figure. However, it is clear that the northern part of the Colosseum is situated on a layer of the Pleistocene continental deposits, while the southern part sits on a much softer material, which is a sedimentary filling of the former tributary of the River Tiber. The estimated shear wave velocities in the Pleistocene continental deposits and tributary sediments are $400 \mathrm{~m} / \mathrm{s}$ and $100-200 \mathrm{~m} / \mathrm{s}$, respectively. Both the layer of the Pleistocene continental deposits and the tributary sediments are underlain by more consolidated Pliocene marly clays with an estimated shear wave velocity of $800 \mathrm{~m} / \mathrm{s}$. The considerable velocity contrast between the tributary sediments and the surrounding material means that the tributary sediments represent a well confined sedimentfilled valley.

Estimated values of the quality factor in the 
Pleistocene continental deposits, valley, and underlying marly clays are 40,7-15, and 100, respectively. Finally, the corresponding density estimates are 1900, 1800, and 2050, all in $\mathrm{kg} / \mathrm{m}^{3}$.

All above values are preliminary and based on the direct in-situ measurements in similar materials at sites outside the Colosseum area.

The valley is relatively deep $-26 \mathrm{~m}$ at the center while only $78 \mathrm{~m}$ wide on the free surface. Its shape ratio (the ratio of the maximum sediment thickness $h$ to a total width over which the thickness is larger than $h / 2$; introduced by Bard and Bouchon, 1985) is 0.47. The steep valley slope means a strong lateral discontinuity between the Pleistocene continental deposits and the tributary sediments. Clearly, the Colosseum siting is very unusual the structure spans over the strong lateral discontinuity.

Because of the uncertainty in shear wave velocity $\beta$ and quality factor $Q$ values in the valley we considered two models: model M1 with $\beta=100 \mathrm{~m} / \mathrm{s}$ and $Q=15$, and model M2 with $\beta=200 \mathrm{~m} / \mathrm{s}$ and $Q=7$. Though lowervelocity materials usually have lower $Q$ we have chosen the opposite rule and constructed two extreme models in order to obtain the upper and lower estimates of the site effect.

\subsection{Method}

We used the finite-difference technique described in Moczo (1989) and Moczo and Bard (1993) to compute the responses of the two models. The spatial variation of the density and shear modulus (internal discontinuities in the case of homogeneous blocks) is accounted for by effective parameters. The effective density is evaluated as an integral arithmetic average of the density in a certain vicinity of a grid point. The effective horizontal and vertical shear moduli are evaluated as integral geometric averages of shear moduli in between two neighboring grid points in the horizontal and vertical directions, respectively. Thus, the actual position of the material discontinuity is taken into account in evaluating the effective parameters. In this sense we follow the smooth shape of the valley boundary shown in fig. 2 . We would like to stress that we use the finitedifference technique in which effective parameters are defined as geometric averages. As shown by Zahradník et al. (1993) and Zahradník (1993) this feature is closely related to the consistency of the finite-difference schemes at material discontinuities.

The model was covered by an irregular spatial grid. The size of the grid spacings allowed us to compute the response up to $15 \mathrm{~Hz}$. The irregular spatial grid made it possible to model the free surface smoothly (i.e. without gridrelated steps) in segments E-A and D-F, see fig. 2. More details on the model, spatial grid, and computation are given in the Appendix.

Both models were excited by a vertically incident plane $S H$ wave. The $S H$ response in both the time and frequency domain was computed at the free surface of the geologic structure.

In order to investigate transfer properties of the geologic structure in various frequency ranges, we simulated different time functions of the incident wave. The «delta-like» impulse with a relatively broad amplitude spectrum as well as the wavelets with narrow amplitude spectra centered around specified frequencies were easy to simulate using the analytic Gabor signal with three parameters. The definition of the Gabor signal, the parameters of five signals I1-I5 used in this study, and the figures of the signals and their amplitude spectra are given in the Appendix.

\section{Numerical results}

\subsection{Pseudoimpulse responses}

Figure 3 shows the pseudoimpulse response, i.e. the time-domain response to a relatively short «delta-like» impulse (I1 signal, see the Appendix), at the free surface of the both models. As expected, there is a striking difference between the surface motion in the A-B and B-C-D segments. In the A-B segment we clearly recognize a synchronous arrival of the direct wave. What follows is a site-dependent superposition of the waves propagating vertically between the free surface and the horizontal 


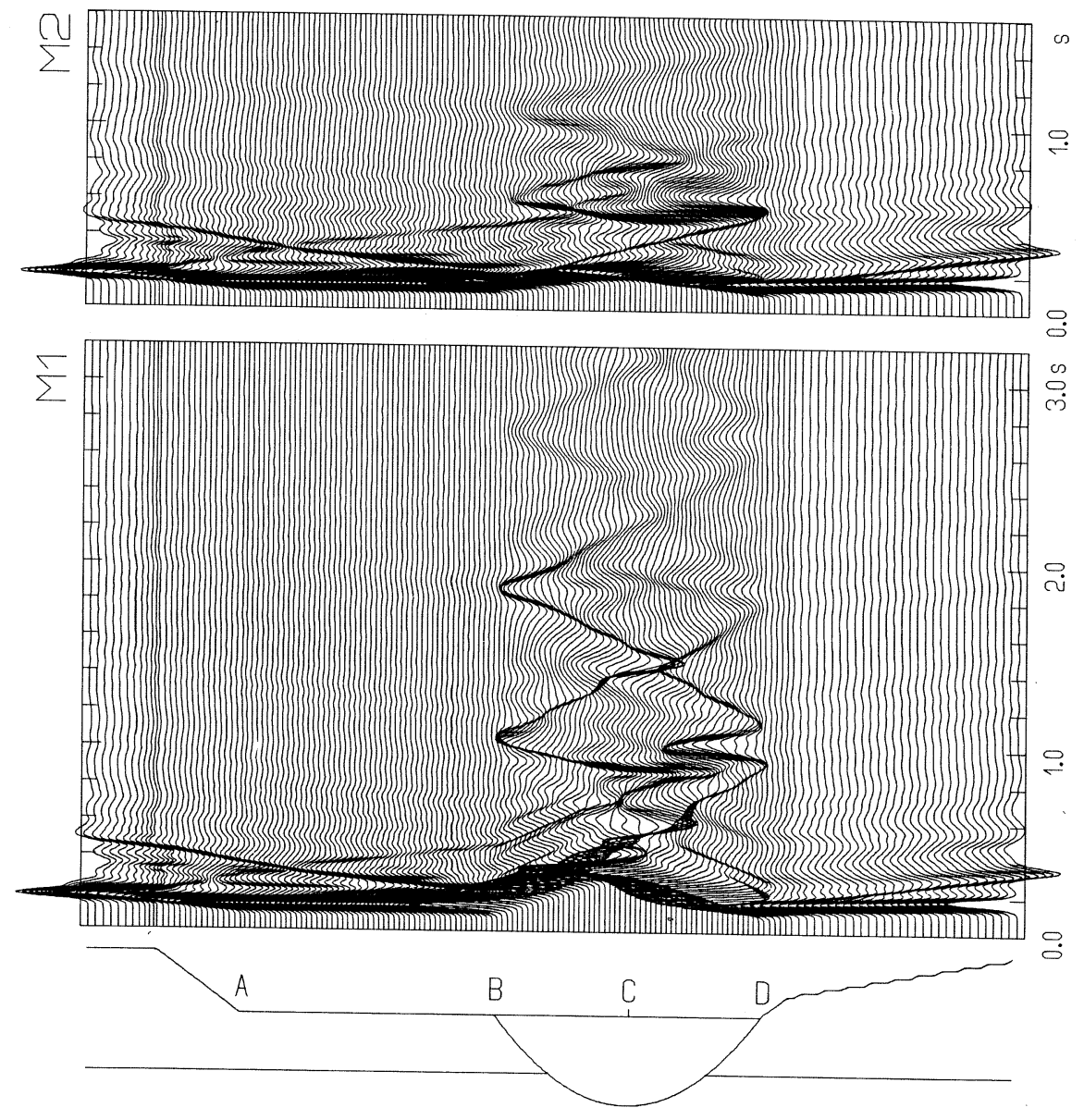

Fig. 3. Pseudoimpulse responses of the M1 and M2 models - time-domain responses to a relatively short «delta-like» I1 signal - at the free surface sites. The irregularity in trace spacing to the left of A edge, and to the right of $\mathrm{D}$ edge is connected with the irregularity of the computational grid. Amplitude scale: $0.8 \mathrm{~cm}$ in the figure corresponds to the actual surface displacement of $2.649 \mathrm{~cm}$ for a maximum basement displacement
of $1 \mathrm{~cm}$.

layer-basement interface, and the laterally propagating waves reflected at and transmitted through the valley slope, and waves diffracted from the E, A, and B edges. Due to a small velocity contrast between the layer and the basement, the effective surface motion has relatively short duration - only 3-4 times longer than the incident impulse.

In both the M1 and M2 models, the valley exhibits a different behavior. We can distinguish two basic types of wave propagation inside the valley. First, a part of energy is vertically bouncing in the central part of the valley. For example, in the M1 model, the peaks at times of 0.86 and $1.38 \mathrm{~s}$ correspond to waves once and twice reflected from the valley bottom, respectively. Second, there are laterally propagating waves from both valley sides. 
They include waves transmitted through the valley slopes and waves diffracted from the B and D edges. Apparently, less energy enters the valley at its right-hand side since the free surface to the right of the margin climbs up preventing thus vertical reverberations in the layer and reflecting the energy to the right. This is the reason why the wavefield inside the valley is not symmetric with respect to the vertical axis at the valley center though the valley itself is. The laterally propagating waves travel between the valley slopes where they are reflected back.

There is one important feature of the wave propagation in the valley: the wave propagating laterally e.g. from the left-hand side arrives at the right-hand side approximately at the same time $(0.84 \mathrm{~s})$ as the wave once vertically reflected from the valley bottom reaches the free surface in the central part of the valley. This is a specific feature which cannot be observed in shallow valleys and indicates that the resonance phenomena involving the whole sediment body may develop.

Obviously, due to a larger velocity and smaller $Q$ inside the valley in the M2 model the valley response exhibits smaller amplitudes. This and shorter propagation times result in a shorter overall duration.

We also computed the pseudoimpulse responses for three simplified modifications of the M1 and M2 models. In all modifications (they will be shown later) the free surface topography is removed and two of them are without the horizontal layer. The responses are simpler but the basic features of the wavefield inside the valley remain.

\subsection{Fourier transfer functions}

In order to obtain information on transfer properties in the frequency domain we computed the Fourier Transfer Functions (FTF) dividing the Fourier spectrum of the local pseudoimpulse response by the spectrum of the I1 signal. This means that the amplitude equals 2 at the zero frequency. Figure 4 shows moduli of the FTF for the flat part of the free surface. As in the case of the pseudoimpulse responses we observe a remarkable difference between the FTF for the flat-layer surface (A-B segment) and the valley surface (B-C-D segment).

The FTF for the A-B segment are relatively flat. For all positions the amplification (with respect to the incident wave) at frequencies below $2 \mathrm{~Hz}$ is about 2 . The maximum amplification of about 5 at the frequency of $5.2 \mathrm{~Hz}$ can be observed in the central part of the A-B segment where the shape of the FTF is relatively close to that for a 1-D model of a layer overlying a halfspace. On the contrary, the FTF for the valley surface sites exhibit sharp peaks and the maximum amplification at the center reaches factor of 21.3 at $1.2 \mathrm{~Hz}$ in the M1 model and 13 at $2.2 \mathrm{~Hz}$ in the M2 model. It is important that in both models the frequency of the first and largest peak $(1.2 \mathrm{~Hz}$ in the M1 model, $2.2 \mathrm{~Hz}$ in the M2 model) does not change along the valley surface despite a varying local thickness of sediments. This is also true about the frequencies of some other peaks $2.4,3.0,3.56,4.2,4.8 \mathrm{~Hz}$ in the M1 model and $4.3,5.7 \mathrm{~Hz}$ in the M2 model. This indicates that corresponding resonance phenomena involve the whole valley. Let us look at the spatial variation of the peak amplifications at these frequencies (fig. 5). We can see that the 1.2, 2.4 and $3.0 \mathrm{~Hz}$ curves for the M1 model show the same type of amplitude variation as the 2.2, 4.3 and $5.7 \mathrm{~Hz}$ curves for the M2 model, respectively, though the amplification level is obviously lower in the M2 model. This indicates the same type of the valley behavior in both models at the corresponding frequencies. Let us recall that the asymmetry in the curves with respect to the valley center (obvious mainly at the higher frequencies) is due to the asymmetry of the valley surroundings.

\subsection{Time-domain responses to signals with narrow amplitude spectra}

We may better understand the valley behavior at specific frequencies by computing the time-domain responses to the signals with relatively narrow amplitude spectra centered 


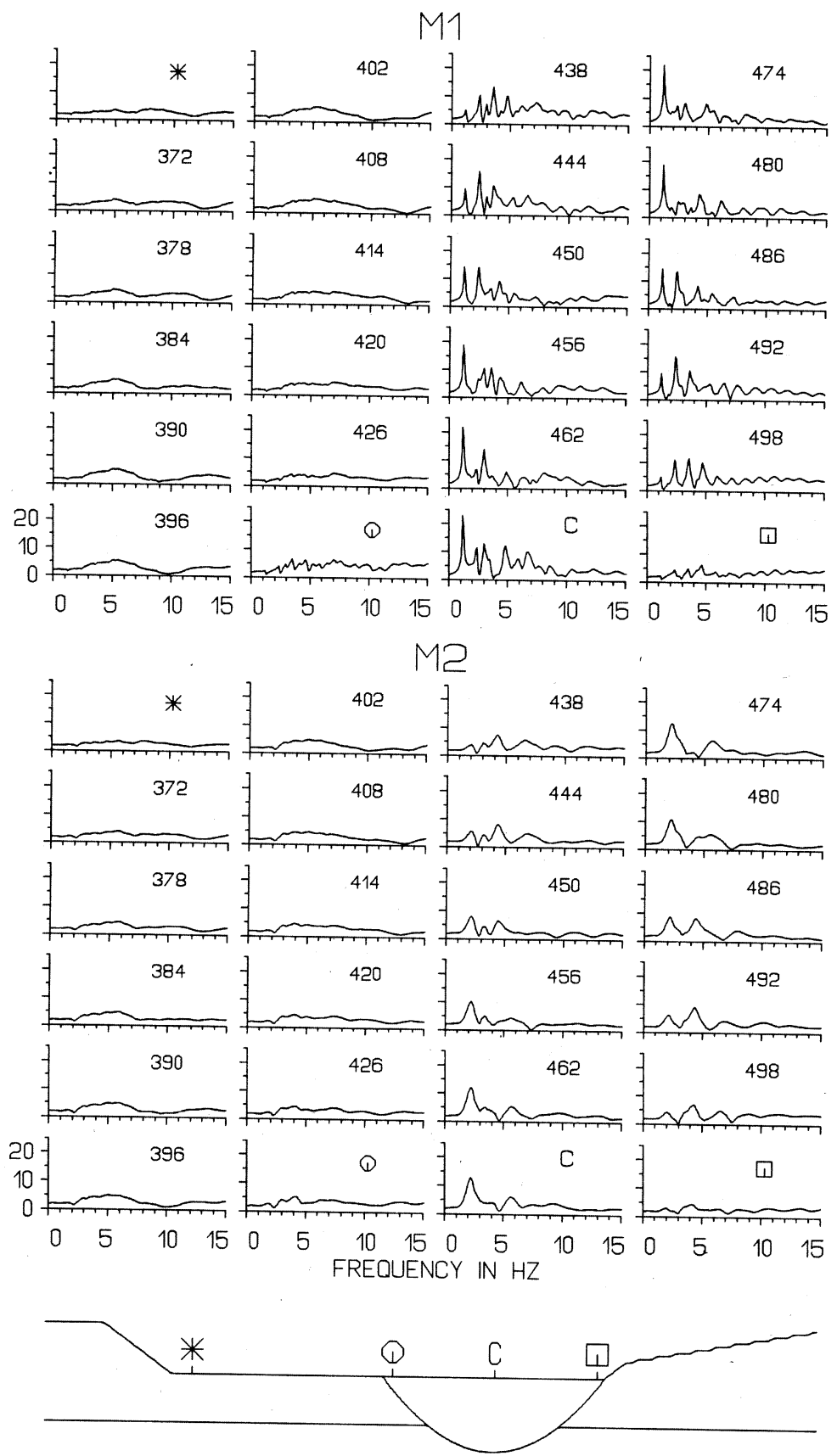

Fig. 4. Fourier Transfer Functions (FTF) for the sites at the flat part of the free surface in the M1 and M2 models. Since they show the spectral amplifications with respect to the incident signal, the amplitude equals 2 at the zero frequency. The sites are indicated by a special symbol or by a horizontal coordinate in meters ac-
cording to fig. 2 . 


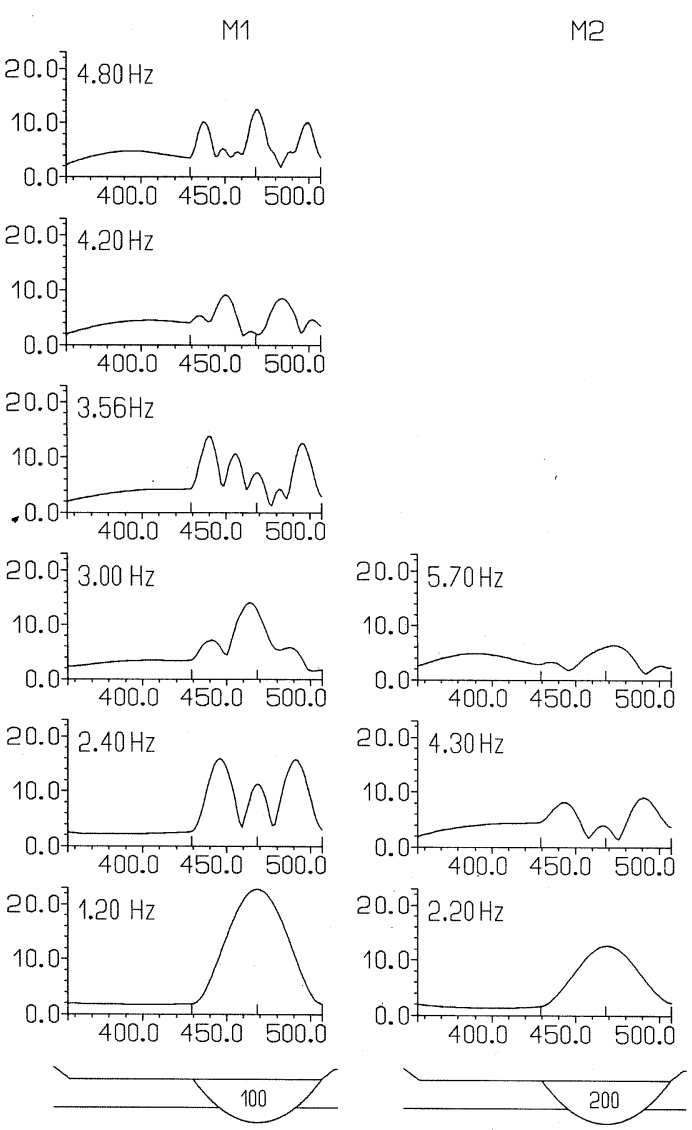

Fig. 5. Spatial variation of the FTF amplitudes at frequencies at which the FTF, shown in fig. 4, exhibit peaks. For example, the left lowermost curve labeled $1.2 \mathrm{~Hz}$ shows the spatial variation of the FTF amplitude at frequency of $1.2 \mathrm{~Hz}$; see the 1.2 $\mathrm{Hz}$ peak in FTF shown in fig. 4.

around these frequencies. The responses of the M1 and M2 models to incoming signals I2 (centered around $1.2 \mathrm{~Hz}$; see the Appendix) and I4 $(2.2 \mathrm{~Hz})$, respectively, are shown in fig. $6 a$. We can see an in-phase motion of a major part of the valley surface. The amplitude is maximum at the valley center and decreases monotonously toward the valley margins similarly to the spectral amplification; see 1.2 and $2.2 \mathrm{~Hz}$ curves in fig. 5 . In both models, the duration of the surface motion in the valley is longer than that in the flat-layer (A-B seg- ment). The relative prolongation is obviously smaller in the M2 model. Considering both the time-domain behavior and the corresponding spectral amplification we recognize the fundamental antiplane shear mode of the 2-D resonance as described by Bard and Bouchon (1985).

Figure $6 \mathrm{~b}$ analogously shows the responses of the M1 and M2 models to the signals I3 (centered around $2.4 \mathrm{~Hz}$ ) and $\mathrm{I} 5(4.3 \mathrm{~Hz}$ ), respectively. Now the resonant pattern is more complicated. There are two nodes located close together and symmetrically with respect to the valley center. The surface motion changes sign at these nodes and is in phase between the valley margins and adjacent nodes and also between the two nodes. Consequently, there are three local maxima along the valley surface; compare with 2.4 and $4.3 \mathrm{~Hz}$ curves in fig. 5. Referring again to Bard and Bouchon (1985) we recognize the first higher symmetric mode of the 2-D resonance.

\subsection{Effect of valley surroundings on the 2-D resonance}

Since we now understand that the first and second peaks in the FTF are due to the 2-D resonance of the valley let us check how the FTF for the valley surface sites depend on the valley surroundings. We may use modifications of the M1 and M2 models which allow us to remove the effects of topography and horizontal surface layer in the medium surrounding the valley. The FTF of the M1 model and its $\mathrm{A}, \mathrm{B}$, and $\mathrm{C}$ modifications are shown in fig. 7 . We can see that the frequencies of all prominent peaks are the same for all models. Comparing M1A and M1B shows that the fundamentalmode resonance peak is almost insensitive to the presence of the $400 \mathrm{~m} / \mathrm{s}$ surface horizontal layer. The twice smaller amplitude in the M1C model confirms that the fundamental-mode amplification is, in the flat-surface and symmetric models, almost fully determined by the velocity contrast between the valley sediments and the basement in the central part of the valley. Then it is interesting to see the effect of the asymmetric topography looking at the M1 

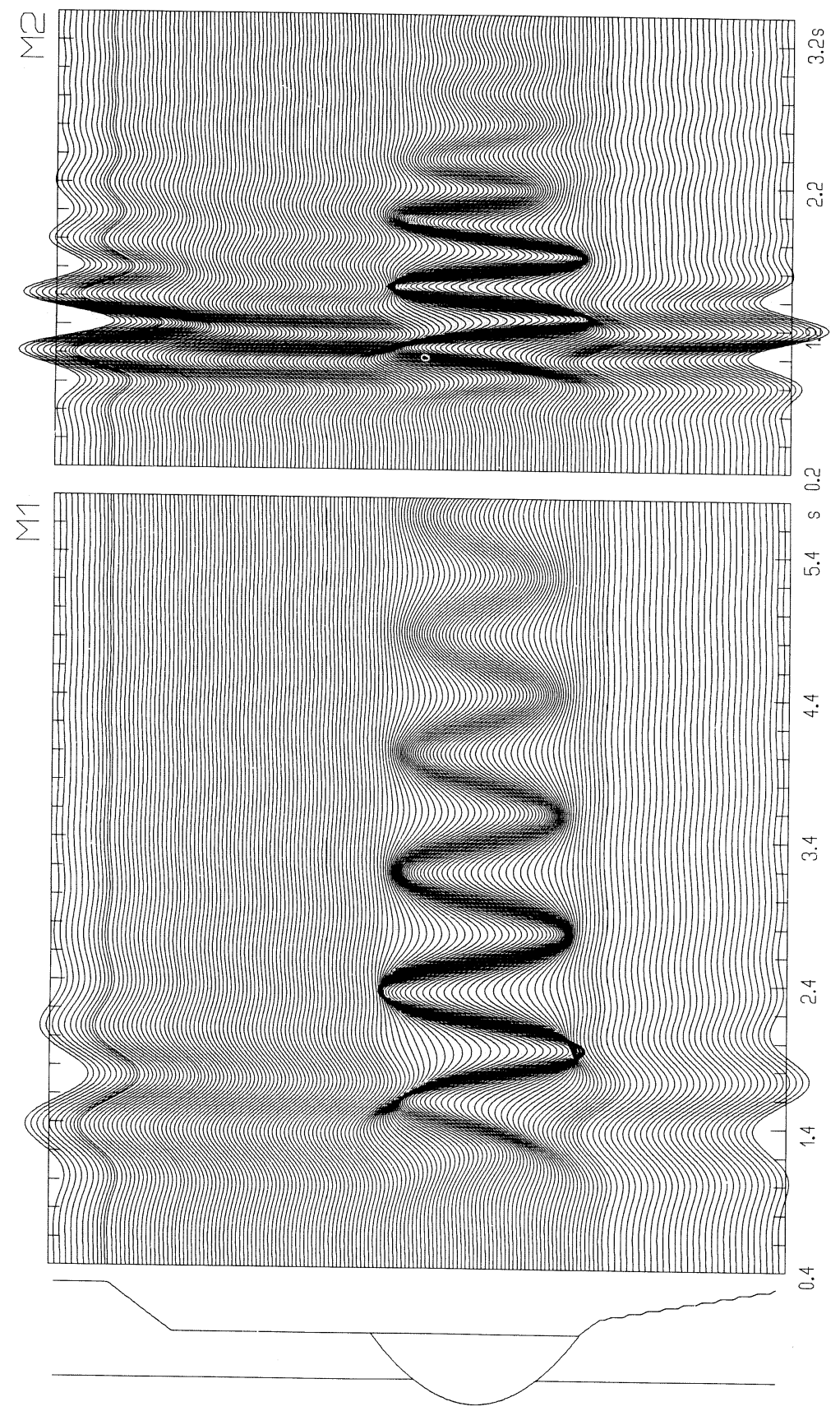

Fig. 6a. Time-domain responses of the M1 and M2 models to the I2 and I4 signals with narrow amplitude spectra centered around frequencies of 1.2 and $2.2 \mathrm{~Hz}$, respectively, at which the FTF in fig. 4 exhibit largest peaks. Time-domain representation of the fundamental antiplane shear mode of the 2-D resonance. Amplitude scale: $0.7 \mathrm{~cm}$ in the figure corresponds to the actual surface displacement of $4.414 \mathrm{~cm}$ for a maximum basement displacement of $1 \mathrm{~cm}$. 

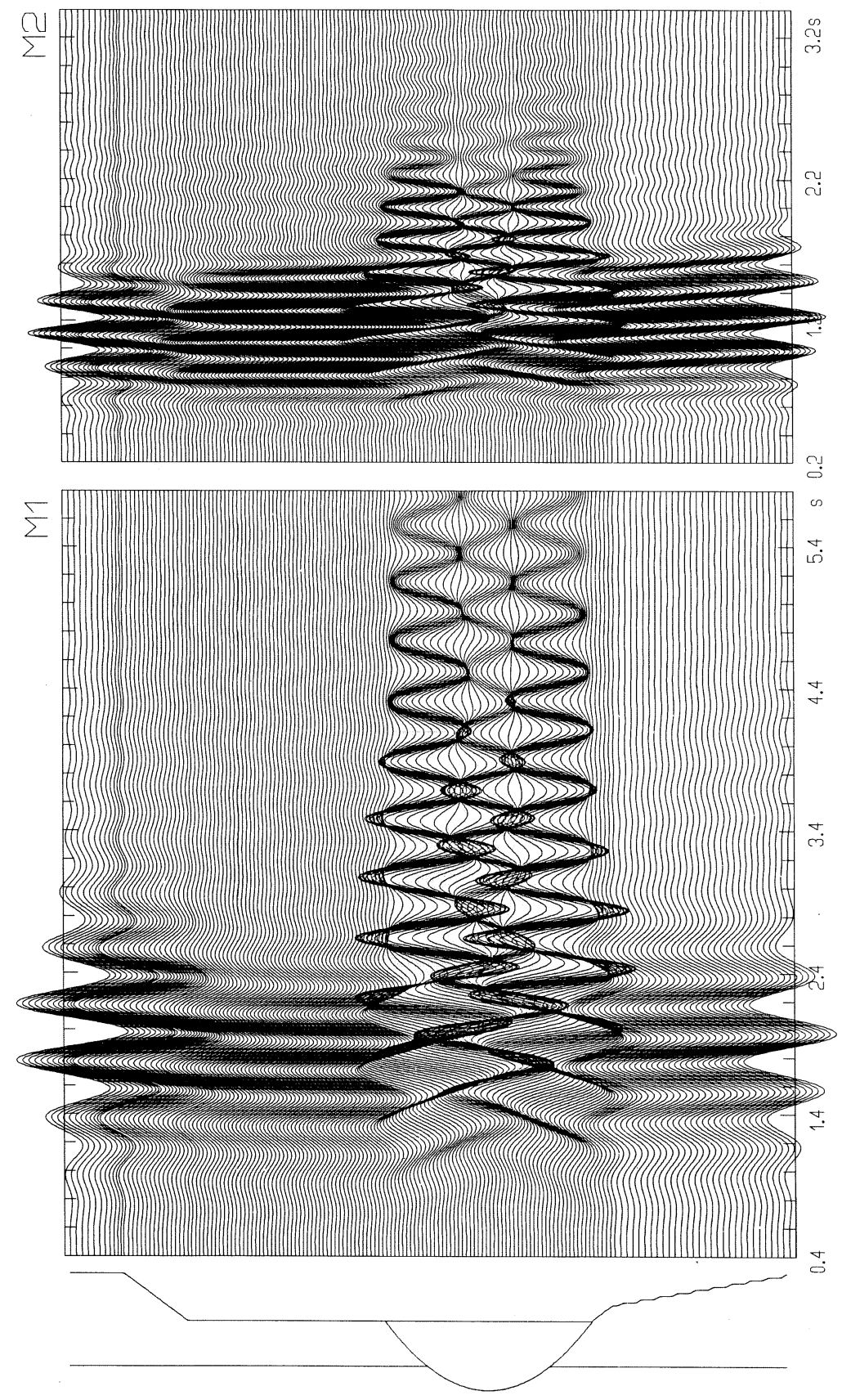

Fig. 6b. Time-domain responses of the M1 and M2 models to the I3 and I5 signals with narrow amplitude spectra centered around frequencies of 2.4 and $4.3 \mathrm{~Hz}$, respectively, at which the FTF in fig. 4 exhibit second largest peaks. Time-domain representation of the first higher symmetric shear mode of the 2-D resonance. Amplitude scale: $0.7 \mathrm{~cm}$ in the figure corresponds to the actual surface displacement of $4.414 \mathrm{~cm}$ for a maximum basement displacement of $1 \mathrm{~cm}$. 


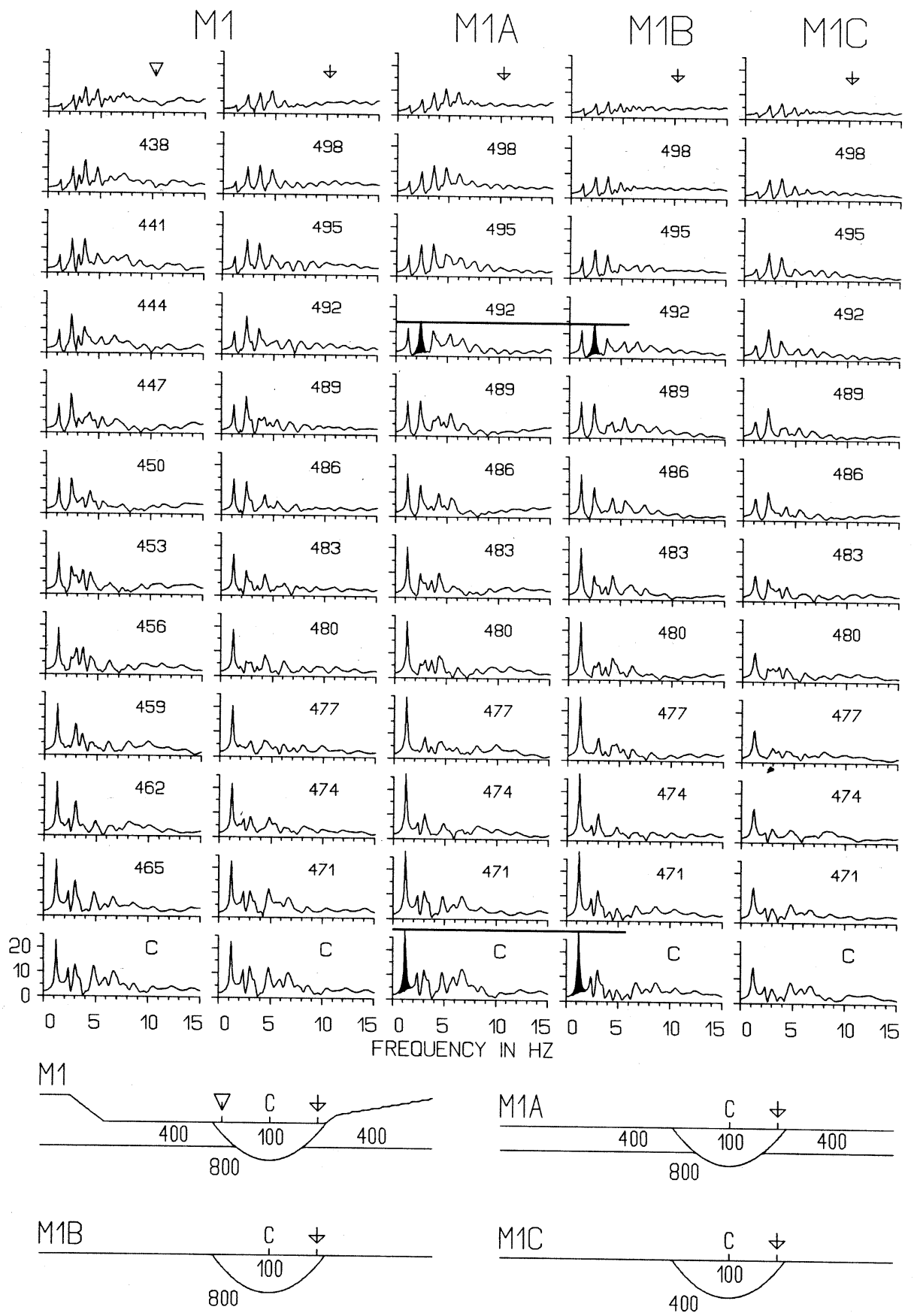

Fig. 7. Fourier transfer functions for the valley surface sites. Since the wavefield inside the valley is symmetric in the M1A, M1B, and M1C models, only the FTF for the right-hand half of the valley surface are displayed. 
model. Apparently, the asymmetric topography has a larger influence on the fundamentalmode amplification than the presence of the $400 \mathrm{~m} / \mathrm{s}$ layer in the flat-surface model.

Comparing the maximum amplifications related to the first higher mode, i.e. the second peak in the FTF for the receiver at $492 \mathrm{~m}$ (and also $444 \mathrm{~m}$ in the M1 model), we see that the amplification is not much sensitive to all model variations. Though the velocity contrast at the valley slopes in the M1B model is twice as large as in the M1A model, the difference between the first higher mode related maximum amplifications in both models is not more than $5 \%$. The amplification in the M1C model is only a little bit smaller. An explanation has to be linked with the fact that, as found by Bard and Bouchon (1985), the first higher mode is mainly due to lateral interferences inside the valley. The small difference between $\mathrm{M} 1 \mathrm{~B}$ and M1C is probably related to small differences in angles and coefficients of transmission from the basement into the valley. The representative value of the angle at which the exciting plane wave incides at the valley slope is approximately 43 degrees. The difference in the transmission angle and transmission coefficient due to change in the basement velocity from $800 \mathrm{~m} / \mathrm{s}$ (in the M1B model) to $400 \mathrm{~m} / \mathrm{s}$ (M1C) is just 4.93 degrees $(=9.82-4.89)$ and $-0.22(=1.52-1.74)$, respectively.

The reason for very close maximum amplifications in the M1A and M1B models may be that the effect of smaller transmission coefficients in the M1A model is compensated by a larger amplitude of the incident wave (after transmission from the basement into the horizontal $400 \mathrm{~m} / \mathrm{s}$ layer) and possibly also by a contribution of the wave reflected upwards from the horizontal interface and entering the valley. It is not obvious, however, at which frequencies this mechanism takes place. Very close maximum amplifications may also be due to the small sensitivity of a large wavelength to the relatively thin horizontal layer.

Another interesting feature in the response of the M1C model is that the maximum amplification related to the first higher mode (at the 489 receiver position) is comparable to that re- lated to the fundamental mode (at the valley center).

Let us look now at the M2 model series. The frequency of the fundamental mode in the $\mathrm{M} 2 \mathrm{~A}$ and $\mathrm{M} 2 \mathrm{~B}$ models remains the same as in the M2 model; see fig. 8. In the M2C model, the velocity contrast along the valley-basement boundary is apparently too small to produce a $2-\mathrm{D}$ resonance in such a way as in all other models under consideration. There is, however, a major peak at the frequency of $2.05 \mathrm{~Hz}$ in the central part of the valley which probably corresponds to a less developed fundamental mode. The frequency of the second peak varies with the model modifications and with the position within the $<4.1,4.35>\mathrm{Hz}$ range.

Comparing the M2 and M2A models we see that, unlike the M1 and M1A models, the asymmetric free-surface topography in the M2 model has apparently a smaller and opposite effect on the maximum amplifications. This indicates that the way how the maximum amplifications are affected is model-dependent and is due to the destructive/constructive interference caused by the asymmetry in the wavefield inside the valley.

Probably more important is, however, what follows from comparing the M2A and M2B models. Similarly as in the case of the M1A and M1B models, the maximum amplification connected with the fundamental mode is not much sensitive to the presence of the horizontal $400 \mathrm{~m} / \mathrm{s}$ layer. There is about $16 \%$ difference between the maximum spectral amplifications connected with the first higher modes in the two models.

\subsection{Differential motion}

The spatial variability of the translational motion over the flat part of the free surface that we observed so far in both the M1 and M2 models indicates the existence of a pronounced differential motion on the valley surface. Figure 9 displays sections of the time-domain differential motion $\partial u / \partial x$ connected with the pseudoimpulse response, and the fundamental and first higher modes of the 2-D resonance in the M1 model. The spatial derivative $\partial u / \partial x$ is 


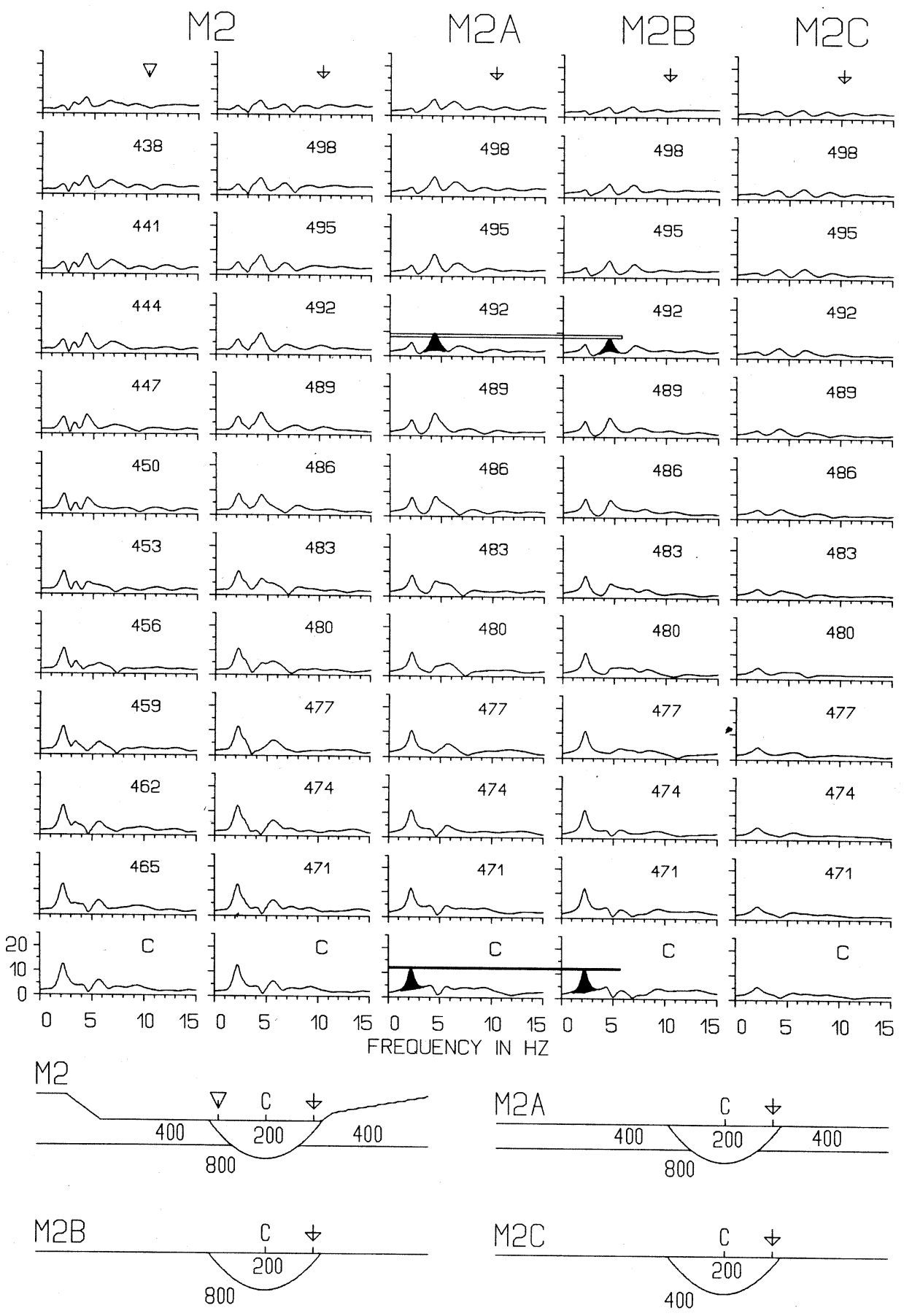

Fig. 8. Similar to fig. 7 but for the M2 model and its modifications. 

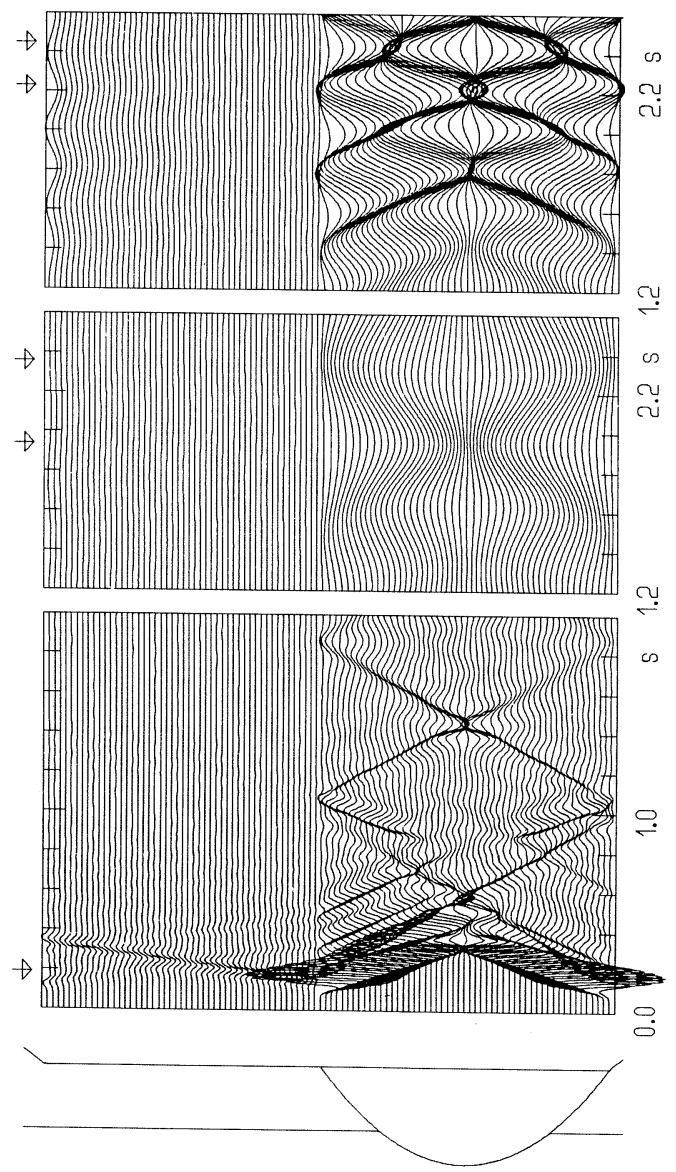

Fig. 9. Surface differential motion $\partial u / \partial x$ in the time domain connected with the pseudoimpulse response (lowermost section), fundamental mode (middle section), and first higher mode (uppermost section) of a 2-D resonance in the M1 model. Amplitude scale: $0.8 \mathrm{~cm}$ in the figure corresponds to the actual surface differential motion of $1.5 * 10^{-2}$ for a maximum basement displacement of $1 \mathrm{~cm}$.

evaluated as a difference between the translational motions at two adjacent positions divided by the corresponding spacing $(0.5 \mathrm{~m})$. The differential motion seismograms for the M2 model (not shown here) have a similar character but a shorter overall duration. As in the case of the translational motion there is a clear difference between the flat-layer surface and valley surface. This is also obvious from fig. 10 where the spatial variations of moduli of the differential motion at specific times (indicated by arrows in fig. 9 in the case of the M1 model) are shown. We see the same characteristic variation pattern related to the fundamental and first higher modes in both models. The M1 model is better for understanding a specific variation pattern in the case of the pseudoimpulse response. A narrow spatial range of the large differential motion is connected with the fact that a relatively very short

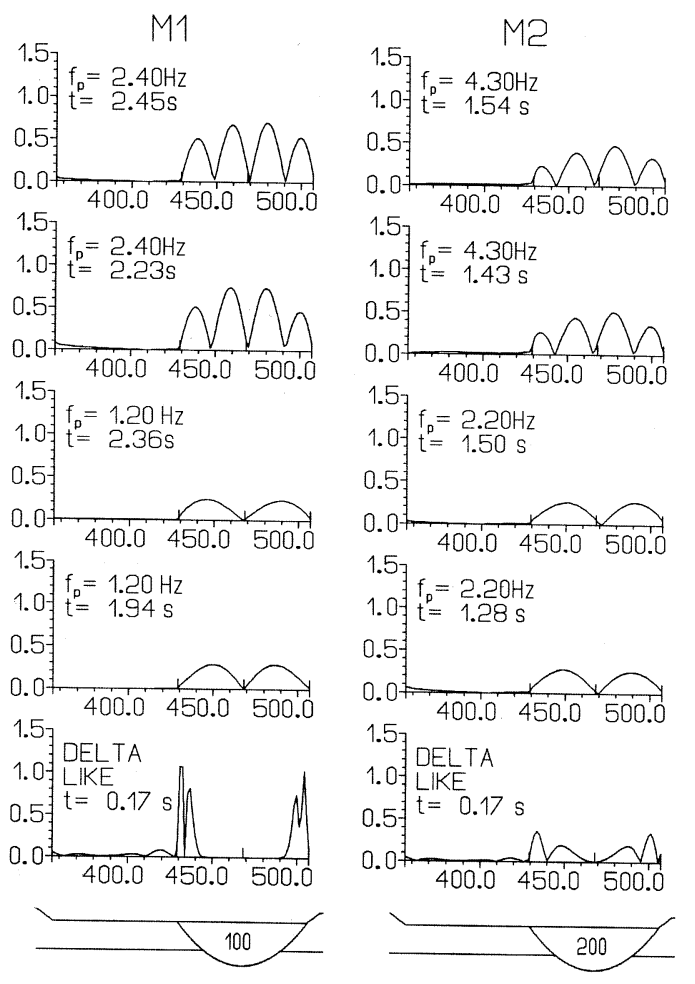

Fig. 10. Spatial variations of the moduli of the time-domain differential motion connected with the pseudoimpulse response (delta-like), fundamental mode $(1.2$ or $2.2 \mathrm{~Hz})$, and first higher mode $(2.4$ or $4.3 \mathrm{~Hz}$ ) of a 2-D resonance in the M1 and M2 models at selected times. The values on the vertical axis have to be multiplied by $10^{-2}$ so that the surface differential motion be scaled for a maximum basement displacement of $1 \mathrm{~cm}$. 
pulse propagates in the slow $(100 \mathrm{~m} / \mathrm{s})$ valley sediments. Two narrow differential motion peaks on both valley sides are due to the propagation of two waves - the refracted wave (incident wave transmitted through the valley slopes) is closely followed by the edge-induced laterally propagating wave. Since the velocity in the M2 model is twice larger, the affected part of the valley surface is approximately twice larger in the M2 model.

The differential motion reaches large values on the valley surface. The spatial variations of the differential motion maxima taken over the entire computed time windows are displayed in fig. 11. For example, the differential motion due to the fundamental mode reaches a significant value of about $3 \cdot 10^{-3}$ for a maximum basement displacement of $1 \mathrm{~cm}$. The differential motion due to the pseudoimpulse response and the first higher mode is even higher. An interesting observation is very close absolute
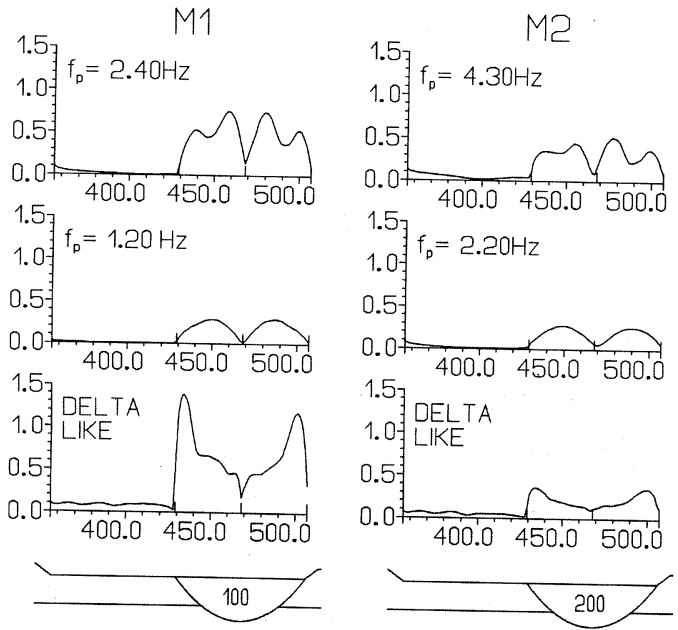

Fig. 11. Spatial variations of the moduli of the differential motion maxima - taken over the entire computed time window - connected with the pseudoimpulse response (delta-like), fundamental mode $(1.2$ or $2.2 \mathrm{~Hz})$, and first higher mode $(2.4$ or 4.3 $\mathrm{Hz}$ ) of the 2-D resonance in the M1 and M2 models. The values on the vertical axis have to be multiplied by $10^{-2}$ so that the surface differential motion be scaled for a maximum basement displacement of $1 \mathrm{~cm}$. values of the differential motions due to the fundamental modes in both the M1 and M2 models (despite the twice smaller velocity contrast in the M2 model) and comparable values in the case of the first higher mode.

\section{Discussion and conclusions}

\subsection{The Colosseum}

The effect of the local geologic structure underlying the Roman Colosseum on a seismic ground motion was investigated. An $\mathrm{SH}$ seismic response was computed assuming a vertical plane-wave incidence. Two models (M1 and M2) were investigated: because of the uncertainty of true model parameters, two sets of the velocity and quality factor values were considered for the same preliminary model geometry.

The computations indicate that the northern and southern parts of the Colosseum may be exposed to significantly different ground motions during earthquakes.

The free-surface ground motion in the southern part exhibits significantly larger amplitudes, differential motion, and longer duration compared to the northern part of the studied profile. The above difference is due to a pronounced lateral heterogeneity beneath the Colosseum. The southern part of the Colosseum is underlain by a relatively deep and soft sediment-filled valley created by the sedimentary filling of the former tributary of the River Tiber. In both studied models a 2-D resonance of the whole valley may develop.

The assumption of a plane-wave incidence in the case of a 2-D resonance is probably not as restrictive as in the case of some other local phenomena. A small sensitivity of the global valley resonance to the source position was observed in extensive experiments by Tucker and King (1984). Development of the resonance involving the whole sedimentary body regardless of a type of valley excitation was theoretically demonstrated e.g. by Bard and Bouchon (1985), Rial and Ling (1992), and Zhou and Dravinski (1994); the incident wave only affects excitation of different modes. 
The present study may be considered as a first step in an effort to understand the effect the local geologic conditions might have had and still may have on the Colosseum during earthquakes.

A geologic and geophysical survey is underway to establish a more detailed and precise model. Future studies with this model should include the $P-S V$ in 2-D and possibly the complete wavefield in 3-D modeling, and realistic excitations. Estimates of the actual motion for historical earthquakes should also be included. Finally, a soil-Colosseum interaction should be accounted for to understand the specific damage pattern.

\subsection{2-D resonance in valleys embedded in heterogeneous medium}

Though preliminary in relation to the Colosseum, the studied models (M1, M2, and their modifications; see figs. 2, 7 and 8) represent an interesting type of local geologic conditions. While in the previous theoretical papers dealing with the 2-D resonance (e.g. Bard and Bouchon, 1985; Rial and Ling, 1992; Zhou and Dravinski, 1994) the valley was embedded either in a homogeneous hardrock or in a rigid medium, we observed the 2-D resonance in the case of considerably heterogeneous valley surroundings.

From this point of view it is interesting to recall the existence condition found by Bard and Bouchon (1985). They presented a criticalshape-ratio curve versus velocity contrast. Points above the curve in their fig. 12 represent valleys with the 2-D resonance being a main phenomenon. Points below the curve represent valleys with the $1-\mathrm{D}$ resonance + lateral propagation of surface waves. Our homogeneousbasement models (M1B, M1C, and M2B; see figs. 7 and 8) with the shape ratio of 0.47 are well above the curve and behave as predicted. One model (M2C) is below the curve but we observe at least a less developed fundamental mode of the 2-D resonance in the central part of the valley (see fig. 8). The four other models (M1, M1A, M2, and M2A) are more com- plicated. They include two different velocity contrasts along the valley-surroundings boundary. For a given shape ratio, 0.47 , all velocity contrasts in the two models (M1 and M1A) are above the curve. Again, the two models behave as predicted.

However, in the two models (M2 and M2A), the velocity contrast of 4 at the valley bottom is well above the curve while the contrast of 2 at the valley slopes is well below the curve. Neither of the two models thus satisfy the above condition for the existence of the 2-D resonance. Nevertheless, we observe the $2-\mathrm{D}$ resonance in both the models.

The maximum spectral amplifications connected with the fundamental and first higher modes of the 2-D resonance are not much sensitive to the presence of the horizontal surface layer (with an intermediate velocity) in the valley surroundings (compare M1A with M1B in fig. 7 and M2A with M2B in fig. 8).

The maximum time-domain differential motion connected with the fundamental mode of the 2-D resonance reaches very close values in two models (M1 and M2; see fig. 11) despite the fact that the velocity and impedance contrasts in one model (M2) are twice smaller than those in the second model (M1).

We think that the above findings on the 2-D resonance in a realistic case deserve more investigations elsewhere.

\section{Acknowledgements}

The idea of Renato Funiciello on a strong geologic heterogeneity beneath the Colosseum motivated this work. Enzo Boschi encouraged authors to proceed in the research. This study was initiated during the visit of one of us (P.M.) to Istituto Nazionale di Geofisica (ING) in Rome. In addition to internal research funds by ING, partial support was provided by the Grant Agency for Science, Slovak Academy of Sciences (Grant No. 2/1064/94.), and by Consiglio Nazionale delle Ricerche, Progetto Strategico Beni Culturali. We want to thank Renato Funiciello and Fabrizio Marra for compiling the geologic model considered in the 
study. We also thank Pierre-Yves Bard, Franta Hron and Jiří Zahradník for the critical reading of the manuscript and valuable comments.

\section{Appendix}

Model and grid - The total cross-sectional area considered in the computation is $982 \mathrm{~m}$ long and $117 \mathrm{~m}$ deep. It is covered with a rectangular $770 \times 147$ grid with irregular spacings in both the horizontal and vertical directions. The minimum grid spacing, $0.5 \mathrm{~m}$, is used in the central flat-free-surface part of the profile.

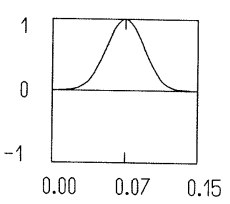

11
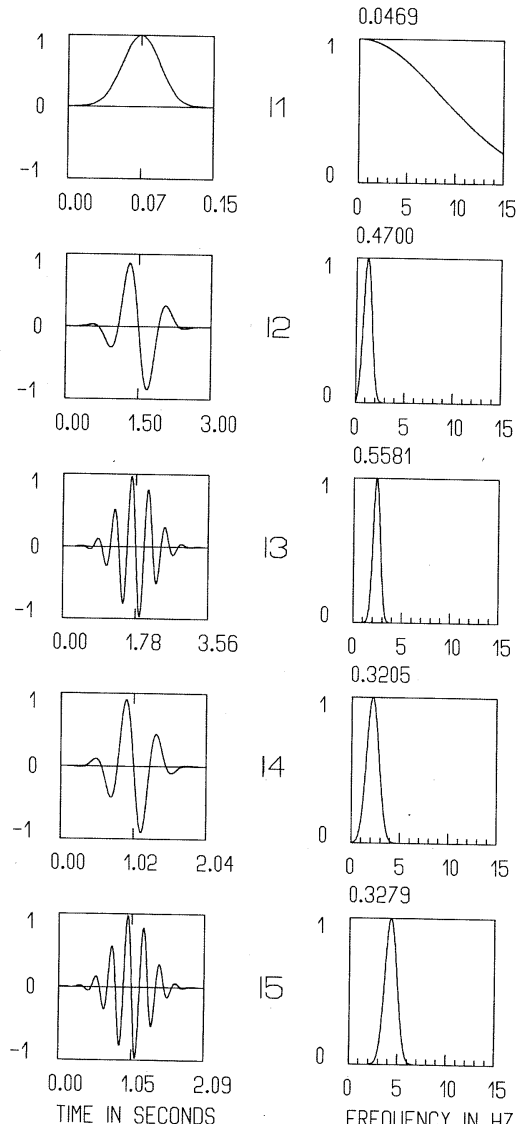

Fig. A1. Input signals used in the study and their amplitude Fourier spectra.
The maximum grid spacing, $2.2 \mathrm{~m}$, is used in the lower part of the basement and close to the left- and right-hand side model margins. The theoretical accuracy of the finite-difference computation is at least up to $15 \mathrm{~Hz}$. The time step of $0.00039 \mathrm{~s}$ was used to compute the time-domain responses. The computed time windows were not shorter than $12 \mathrm{~s}$. The Reynolds (1978) formulae were used to simulate transparent boundaries of the computational grid.

Input signals - A time function of the incident plane $S H$ wave is given by a Gabor signal defined by

$$
s(t)=e^{-\left(\omega_{p}\left(t-t_{s}\right) / \gamma\right)^{2}} \cos \left(\omega_{\rho}\left(t-t_{s}\right)+\psi\right),
$$

with

$$
\omega_{p}=2 \pi f_{p}, t_{s}=0.45 \gamma / f_{p}, \text { and } t \in<0,2 t_{s}>.
$$

Choosing appropriate values of the parameters $\omega_{p}, \gamma$, and $\psi$ makes it possible to obtain signals with relatively broad or narrow amplitude spectra. Five specific signals, referred to as I1 to $\mathrm{I} 5$ in the study, are shown in fig. A1.

\section{REFERENCES}

BARD, P.-Y. and M. BouCHON (1980): The seismic response of sediment-filled valleys. Part 1 . The case of incident SH waves, Bull. Seismol. Soc. Am., 70, 1263-1286.

BARD, P.-Y. and M. BouCHON (1985): The two-dimensional resonance of sediment-filled valleys, Bull. Seismol. Soc. Am., 75, 519-541.

Frankel, A. (1993): Three-dimensional simulations of ground motions in the San Bernardino Valley, California, for hypothetical earthquakes on the San Andreas fault, Bull. Seismol. Soc. Am., 83, 1020-1041.

Frankel, A., S. Hough, P. Friberg and R. Busby (1991): Observations of Loma Prieta aftershocks from a dense array in Sunnyvale, California, Bull. Seismol. Soc. Am., 81, 1900-1922.

Funiciello, R., L. Lombardi, F. Marra and M. PAROTTO (1995): Seismic damage and geological heterogeneity in Rome's Colosseum area: are they related?, Annali di Geofisica, 38, 927-937 (this volume).

Kagawa, T., S. SAwAdA and Y. IwASAKI (1992): On the relationship between dependency of earthquake ground motion and deep basin structure beneath the Osaka plain, J. Physics Earth, 40-1, 73-83.

KING, J.L. and B.E. TUCKER (1984): Observed variations 
of earthquake motion across a sediment-filled valley, Bull. Seismol. Soc. Am., 74, 137-151.

Moczo, P. (1989): Finite-difference technique for $\mathrm{SH}$ waves in 2-D media using irregular grids - application to the seismic response problem, Geophys. J. Int., 99, 321-329.

Moczo, P. and P.-Y. BARD (1993): Wave diffraction, amplification and differential motion near strong lateral discontinuities, Bull. Seismol. Soc. Am., 83, 85-106.

Molin, D. and E. Guidoboni (1989): Effetto fonti, effetto monumenti a Roma: i terremoti dell'antichità a oggi, in I terremoti prima del Mille in Italia e nell'area Mediterranea. Storia archeologia sismologia, edited by E. Guidoboni (SGA, Bologna, Italy), 194-223.

Pantosti, D., G. D’Addezio, F.R. Cinti, D.P. Schwartz and J.C. HAMILTON (1992): Evidence for a prehistoric surface faulting earthquake on the Ovindoli-Pezza fault, Central Italy, EOS Trans. Am. Geophys. U., 73, 353 (abstract).

Phillips, W.S., S. Kinoshita and H. Fujiwara (1993): Basin-induced Love waves observed using the strongmotion array at Fuchu, Japan, Bull. Seismol. Soc. Am., 83, 65-84.

REYNOLDS, A.C. (1978): Boundary conditions for the nu- merical solution of wave propagation problems, Geophysics, 43, 1099-1110.

RiAL, J.A. and H. LING (1992): Theoretical estimation of the eigenfrequencies of 2-D resonant sedimentary basins: numerical computations and analytic approximations to the elastic problem, Bull. Seismol. Soc. Am., 82, 2350-2367.

SEO, K. and H. Kobayashi (1980): On the rather longperiod earthquake ground motions due to deep ground structures of Tokyo area, in Proceedings 7 th World Conference Earthquake Engineering, Istanbul, vol. 1 9-16.

TuCKER, B.E. and J.L. KING (1984): Dependence of sediment-filled valley response on input amplitude and valley properties, Bull. Seismol. Soc. Am., 74, 153-165.

ZAHRADNÍK, J. (1993): Simple elastic finite-difference scheme, Bull. Seismol. Soc. Am. (submitted).

Zahradník, J., P. Moczo and F. Hron (1993): Testing four elastic finite-difference schemes for behaviour at discontinuities, Bull. Seismol. Soc. Am., 83, 107-129.

ZHOU, T. and M. DrAVINSKI (1994): Resonance prediction of deep sediment valleys through an eigenvalue method, Geophys. J. Int., 117, 749-762. 